\title{
Review of: "Alkali Can Accelerate The Cellulose Dissolution In Aqueous 1-Ethyl-3-Methylimidazole Acetate (EmimAc/10\% Water)"
}

\author{
Kaarlo Nieminen ${ }^{1}$
}

1 Aalto University

Potential competing interests: The author(s) declared that no potential competing interests exist.

Review of:" Alkali Can Accelerate the Cellulose Dissolution in Aqueous 1-Ethyl-3Methylimidazole Acetate (EmimAC/10\% Water)"

The study approaches the dissolution of cellulose in ionic liquids with the idea of promoting the dissolution in aqueous EmimAC by adding $\mathrm{NaOH}$. Although the study focuses on experimental work, the authors also discuss possible mechanisms behind the experimental observations. The results confirm that $\mathrm{NaOH}$ increases the cellulose dissolution with decrease in the degree of polymerization as an undesired side effect.

The topic of the study is relevant, and the results are of some interest. Furthermore, the structure of the manuscript is suitable for a scientific article. Moreover, the methods and the conclusions seem to be scientifically sound. However, the presentation of the results should be more comprehensive and explanatory, without assuming the readers already to be familiar with the data and the conclusions. As for English language, the quality is not poor, but the text still needs careful editing to remove elementary spelling and grammatical errors. As an example, there are some occasions of missing present tense third person verb ending s. More importantly, some formulations are either unidiomatic or obscure and must be modified.

Line 16: The word pair potential and successful is almost self-contradictory; if something is potential (= promising) it is still waiting for a breakthrough. Maybe it would be better to change the word order; the IL:S are already successful and they have even further potential. Alternatively, perhaps the intention is to say that IL:S are potent (= strong) solvents.

Line 16: ...cellulose solvents...

Line 25: ...causes...

Line 46: A bit confusing to use the word viscose (usually referring to a type of rayon fabric) in this way. Should it be viscous solution?

Line 48: ....is another obstacle... 
Lines 58-61: Something wrong with this sentence leaving the message nebulous. Whereas is probably not the correct beginning word. Whereas is a conjunction, typically situated in the middle of a sentence, indicating a contrast or comparison within the sentence. (Exothermic processes release energy, whereas endothermic processes absorb it.) This passage seems to contain the essential piece of information that the solubility of cellulose in ionic liquid decreases with (increasing) water content, but the unfortunate formulation suppresses it from the reader.

Line 66: This is a bit unclear. Should the word order be different? ..., instead of plain water, aqueous $\mathrm{NaOH} \ldots$

Line 69: ... less costly...

Line 87: ...complete dissolution...

Line 129: With $2 \%$, do you mean all the $2 \mathrm{wt} \%$ present in the solution (line 82 ) or $2 \%$ of the available cellulose. Even if something is obvious for the authors, it is still important to make the effort to carefully explain it to the reader.

Line 132: Here the word Besides is rather informal - better use e.g. Moreover. Furthermore, what new information does the Besides sentence bring? You already concluded that $\mathrm{NaOH}$ reduces the dissolution time. As for interpunctuation, a semicolon separates two independent clauses, but here the while clause is dependent, hence a comma is the appropriate punctuation mark.

Line 135: ...comprises two models...

Line 142: ...predicted...

Table 1: What is the unit in the third column?

Line 203: ....implies a stronger interaction...

Lines 205-206: Where do the values 3wt\% and 20\% come from? Are they experimental or literature values?

Line 207: ....in the literature...

Line 246: What do you mean by destruction between cations and anions?

Line 258: ... much more obvious... The word more indicates comparison, much only enhances the comparison. Furthermore, obvious is an adjective, whereas obviously is an adverb.

Line 300: ... will act with water... No ending $s$ in the future tense.

Line 316: .... it is certain...

Line 319: ... might indicate another possibility, that is, $\mathrm{NaOH}$ addition causing a conversion...

Line 320: ... much more efficient...

Line 321: prediction

Line 322: requires

Line 362: In this reference you provide the given names with the family names as an initial. In most European languages the formal convention is the other way round so that the listed names would become Marekha B, Bria M, Moreau M etc.

Supporting Information 
Line 55: See previous comment. 